\title{
DECONSTRUCTING ADAPTIVE MANAGEMENT: CRITERIA FOR APPLICATIONS TO ENVIRONMENTAL MANAGEMENT
}

\author{
R. Gregory, ${ }^{1,4}$ D. Ohlson, ${ }^{2}$ and J. Arvai ${ }^{1,3}$ \\ ${ }^{1}$ Decision Research, 1160 Devina Drive, Galiano, British Columbia V0N 1 P0 Canada \\ ${ }^{2}$ Compass Resource Management, Suite 200, 1260 Hamilton Street, Vancouver, British Columbia V6B 2S8 Canada \\ ${ }^{3}$ Department of CARRS, 305 Natural Resources Building, Michigan State University, East Lansing, Michigan 48824 USA
}

\begin{abstract}
The concept of adaptive management has, for many ecologists, become a foundation of effective environmental management for initiatives characterized by high levels of ecological uncertainty. Yet problems associated with its application are legendary, and many of the initiatives promoted as examples of adaptive management appear to lack essential characteristics of the approach. In this paper we propose explicit criteria for helping managers and decision makers to determine the appropriateness of either passive or active adaptivemanagement strategies as a response to ecological uncertainty in environmental management. Four categories of criteria-dealing with spatial and temporal scale, dimensions of uncertainty, the evaluation of costs and benefits, and institutional and stakeholder supportare defined and applied using hypothetical yet realistic case-study scenarios that illustrate a range of environmental management problems. We conclude that many of the issues facing adaptive management may have less to do with the approach itself than with the indiscriminate choice of contexts within which it is now applied.
\end{abstract}

Key words: adaptive-management criteria; decision analysis; decision making; environmental management.

\section{INTRODUCTION}

Few concepts in environmental management are both as widely promoted and as widely misunderstood as adaptive management (AM). Since its inception more than two decades ago (Holling 1978, Walters 1986), AM has been elevated to a position at the forefront of ecological science and environmental management for dealing with problems characterized by high levels of uncertainty (Gunderson and Holling 2002). Yet its track record of implementation is weak (Walters 1997) and many ecological planning, restoration, and recovery initiatives that are promoted under the banner of adaptive management exhibit few, if any, of the characteristics generally considered to be essential. As a result, an overview of the applications literature tells a conflicting story; one could conclude that adaptive management should either be relied upon heavily or criticized sharply when considering solutions to challenging resource management problems.

Much of the conflict can be traced to the appealing nature of AM as a theoretical construct. The central premise of learning by doing is so attractive and universally intuitive that one is hard pressed to find an environmental resource management plan or statement of environmental policy that does not make at least some form of commitment to the use of AM. The problem is that "learning by doing" is often invoked as a management objective without a clear definition of what

Manuscript received 31 August 2005; revised 11 April 2006; accepted 17 April 2006. Corresponding Editor: J. A. Logan.

${ }^{4}$ E-mail: rgregory@interchange.ubc.ca it means (i.e., What constitutes learning and how much of it is required?) or how to properly do it (i.e., How to implement AM?). As a result, AM as applied in many management contexts retains little meaning.

Another source of conflict stems from the urgent need for tools to help environmental management professionals make decisions under conditions of uncertainty. The pairing of significant uncertainty about the behavior and response of ecological systems with urgent calls for near-term action constitutes a difficult reality, and a common lament, for many resource managers. Few are well versed in the science of decision making under uncertainty, so the apparent availability of a method ostensibly designed for just this purpose is understandably appealing.

But there is a dark side to this attraction, for two different groups of environmental-management professionals and for very different reasons. A first group, composed mainly of upper-level managers and bureaucrats who work in environmental-management agencies, is attracted to AM because they seek an accepted approach that can allow for postponement of the difficult decisions that need to be made (often later, and by someone else) in the face of resource constraints and scientific uncertainty. The second group, composed of biological scientists, is attracted to AM because it provides a tenable mechanism for applying the scientific method to challenging problems facing complex ecosystems, often resulting in the design of costly experiments that tend to ignore impacts on other important environmental, social, or economic objectives. 
In this paper we attempt to help untangle some of these conflicts by providing guidance on when AM approaches should be used and, perhaps more importantly, when they should not. In doing so, we present criteria for the selection and design of AM initiatives and evaluate them in the context of several specific environmental-management options drawn from illustrative-hypothetical yet realistic - case-study scenarios in forestry and fisheries management as well as land-use planning.

\section{Implementing Adaptive Management: An Overview}

$\mathrm{AM}$ is designed primarily to help managers learn about complex ecological systems by monitoring the results of a suite of management initiatives. In this sense, it is a systematic approach to improving the management process and accommodating change by learning from the outcomes of a set of environmental management policies and practices (Holling 1978, Walters 1986). The generally stated goal of AM is to improve managers' knowledge about a set of well-defined ecological objectives through the implementation of carefully designed, quasi-experimental management interventions and monitoring programs. At least in theory, the increased knowledge should also assist resource managers in responding to the inevitable ecological surprises that arise over the course of a management intervention (Clark 1980). However, as the later text will discuss in more detail, economic and social and political surprises also can arise over the course of a management intervention, thus creating problems for an ecologically focused adaptive management plan.

Both the theory and the practice of adaptive management have expanded greatly over the past quarter century. AM first emerged from a desire to address practical problems of environmental and natural resources management. The early efforts of Holling, Walters, and their colleagues made progress toward the goal of improved ecosystem understanding through development of theory and quantitative techniques supporting adaptive management. Later efforts by a variety of researchers, summarized in collections edited by Gunderson et al. (1995) and Gunderson and Holling (2002), provided both a useful perspective on ecological science and guidance toward developing improved institutional support for adaptive management.

Two primary types of adaptive management have been defined, "passive" and "active", which vary in their degree of scientific rigor and experimental design (Walters and Holling 1990, Halbert 1993). Both approaches are valuable and (as discussed in more detail in the next sections) either may be considered more or less appropriate depending on the circumstances of a given management problem.

In passive adaptive management, managers typically use historical data, from the specific area under consideration or from areas considered to be ecologically comparable, to develop a "best guess" hypothesis and to implement a preferred course of action. Outcomes are monitored and new information is used to update the historical data set and, if necessary, the hypotheses and management action. This makes good sense when there is high confidence in the anticipated ecosystem response (i.e., the basic structural models are well defined) and managers can focus on refining parameter estimates. Passive adaptive management also makes good sense when the regulatory or institutional constraints are strong, so that the range of possible variations (e.g., in water flows) is small. In practice, unfortunately, passive adaptive management often turns into basic trial and error learning in which explicit hypotheses are absent or vague, the updating of historical data is haphazard, monitoring is incomplete, and only incremental changes are made to monitoring plans. Although learning may occur, the pace is relatively slow and typically without clear implications for management practices. There is also a very real potential for error, because complex interactions and cumulative effects may confound results and analyses. In the worst case, there may be a complete lack of data updating along with no essential changes to management actions (Folke et al. 2004).

Under active adaptive management, in comparison, managers typically seek to define competing hypotheses about the impact of management activities on ecosystem functions and, in turn, design management experiments to test them. In this way, systems are deliberately tested through management interventions, often with several alternative types of management activities attempted in sequence or in parallel so as to observe and compare results. Thus, the scope of an active AM initiative, as conventionally interpreted, can vary from that of a broad, organizing framework for management of a natural environment to a more limited scope that addresses a specific management problem or even one aspect of a problem (Gregory et al. 2006). Either way, the attraction of an active (as opposed to passive) AM approach is that it will, in theory, deliver more statistically testable information in a shorter period of time. However, active approaches are only as good as their experimental design and they require more (often substantially more) resources to plan, implement, and monitor; these higher costs can exceed the capacity of some management agencies (see Gregory et al. 2005). In some cases, active approaches also may involve greater risks to sensitive species or other values, along with a greater willingness and capacity on the part of managers to act on new information.

Both passive and active AM have been applied to ecosystem management problems with varying spatial scales, ecosystem types, ownership patterns, socioeconomic characteristics, risk implications, and political, regulatory, and jurisdictional complexity. This diversity is both an indication of the enthusiasm with which the concept has been greeted and a source of difficulty in that AM, by design, cannot be a "one size fits all" 
solution to complex problems in environmental management that exhibit ecological uncertainty.

Some applications of AM have been relatively simple and small scale. The British Columbia Forest Service, for example, conducted experiments throughout the 1990 s to evaluate alternative forest-harvesting techniques (Taylor et al. 1997). These studies had limited physical impacts beyond the specific treatment location (i.e., they posed no threat to the viability of the overall ecosystem or any individual species) and results were rarely applied to other sites. In contrast, the application of AM approaches to the Florida Everglades (Walters et al. 1992) or the Columbia River Basin (Lee 1992) has been far more complex. In the latter instance, the AM experiments affected multiple interests (farmers, industry, fishers, First Nations/Native Americans), seriously interfered with some local economic activities, required cooperation from multiple regulatory agencies, and had the potential to gravely threaten endangered salmon stocks. In this multi-faceted environment, many of the potential benefits of an AM approach were never realized and, according to at least some participants, many of the critical questions that faced the Northwest Power Planning Council in 1984 when it adopted the AM concept were left unresolved (Lee 1993).

These are legitimate examples of AM. Other cases share little more than the same name. Walters (1997) noted that of 25 major planning exercises for adaptive management that he has participated in, only seven resulted in experiments of an appropriate scale, and only two could be considered well planned in terms of statistical design. Other initiatives, according to Walters (1997:3), have either "vanished with no visible product" or become "trapped in an apparently endless process of model development and refinement."

There have been lengthy discussions about why AM has not been more widely adopted and, when it has, why some applications of AM have proven to be more successful than others. In response to both questions, some authors have suggested that AM is most feasible and most likely to be successful when the application context is small and relatively simple, so that only few regulatory bodies are involved, the number of interest groups is small and the impacts on them are not severe, and the risk to any species is low (McConnaha and Paquet 1996). These comments are helpful but ignore the fact that (as in the Columbia River example) some of the management contexts where help to deal with scientific uncertainty is most needed are undeniably large and complex and messy. Other observers (e.g., Lee 1993, Gregory and Failing 2002) have linked the limited implementation success of AM to some of the strong emotional responses that arise among participants during planning and implementation. Scientists, for example, can become frustrated by the lack of support from policy makers and managers who are impatient with the long time periods that may be required for acquiring statistically valid field trial results. Conversely, administrators can become frustrated by scientists who appear to be insensitive to the risks posed by experimentation and seem to believe that the pursuit of scientific knowledge is a justified end in itself. Meanwhile the public, seeking near-term results and an assurance of success, is often put off by the dual concepts of uncertainty and experimentation, particularly if some of the less successful trials (i.e., those that could "fail") might occur in their own backyard.

Many other reasons have been cited among the difficulties in implementation of AM plans; as in the examples noted above, these reasons stem from a variety of issues related to the often-conflicting priorities of decision makers and scientists, overlapping jurisdictions, stakeholder fears, and ecosystem considerations. (Halbert 1993, McLain and Lee 1996, Walters 1997, Rogers 1998). These reasons include:

- a failure of scientists to understand the broader array of management priorities and to recognize the need to provide information that can be directly used by decision makers;

- the failure by overlapping management agencies to fully and clearly define their responsibilities for implementing an AM plan;

- a lack of emphasis or attention to the processes required for building shared understanding and shared decision making among diverse stakeholders; and

- the tendency among many scientists to overstate their capability to measure complex functional relationships through experimentation.

There is no single response to these diverse concerns. Anecdotal evidence can be found for patiently working within the system, helping to educate and establish an improved dialogue among managers and scientists and the public over time; other evidence points to the benefits of a more "go-it-alone" strategy, particularly among scientists who are frustrated by not being allowed to pursue favored management or treatment options. Some proponents of AM have sought help in improved evaluation techniques, noting in particular the advantages associated with integrating formal decisionanalysis techniques into adaptive management. For example, Peterman and Peters (1998) describe how decision analysis is particularly effective during the planning stage of an active adaptive-management proposal because it can help to compare the expected performance of alternative experimental designs. Other authors demonstrate the use of decision-analysis techniques to calculate the present value of alternative AM strategies (Walters and Green 1997) or show how decision analysis can be used to compare the potential economic performance of experimental and nonexperimental strategies (Sainsbury 1991). And most recently, Failing et al. (2004) demonstrate the integrated use of probabilistic expert judgments and structured multistakeholder decision methods to select and evaluate a preferred AM design. 
TABLE 1. Summary of proposed criteria for deciding whether to use adaptive management (AM), by topic area and formulated as questions.

\begin{tabular}{|c|c|}
\hline Topic-area consideration & Criteria questions \\
\hline \multicolumn{2}{|l|}{ Spatial and temporal scale } \\
\hline Duration & $\begin{array}{l}\text { Is the project timeline to obtain verified results compatible with management } \\
\text { decision-making requirements? }\end{array}$ \\
\hline Spatial extent and complexity & $\begin{array}{l}\text { If spatial extent or complexity is large, are there opportunities to apply AM on a } \\
\text { subset of the problem and scale up? }\end{array}$ \\
\hline External effects & $\begin{array}{l}\text { Have potential issues related to background trends and cumulative effects of } \\
\text { management actions been addressed in the AM design? }\end{array}$ \\
\hline \multicolumn{2}{|l|}{ Dimensions of uncertainty } \\
\hline Parameter uncertainty & $\begin{array}{l}\text { Has the AM design been pared down to focus on only those uncertainties most } \\
\text { likely to influence management decisions? }\end{array}$ \\
\hline Structural uncertainty & $\begin{array}{l}\text { Are there profound structural uncertainties? If so, how will surprise outcomes be } \\
\text { managed? }\end{array}$ \\
\hline Stochastic uncertainty & $\begin{array}{l}\text { How do low-probability random natural and other causal events affect the AM } \\
\text { design and expected outcomes? }\end{array}$ \\
\hline Confidence in assessments & $\begin{array}{l}\text { If the confidence in the proposed AM design is low, can expert judgment or other } \\
\text { techniques help? }\end{array}$ \\
\hline \multicolumn{2}{|l|}{ Costs, benefits, and risks } \\
\hline Specifying benefits and costs & $\begin{array}{l}\text { Can all the costs and benefits (and risks) be documented and communicated in a } \\
\text { manner understandable to all stakeholders? }\end{array}$ \\
\hline Magnitude of effects & $\begin{array}{l}\text { Will the information collected through AM have sufficient predictive ability to } \\
\text { make a difference to managers? }\end{array}$ \\
\hline Multiple objectives & $\begin{array}{l}\text { Does the design and assessment of AM plans explicitly address the multiple goals of } \\
\text { stakeholders (rather than only scientists)? }\end{array}$ \\
\hline Perceived risks of failure & $\begin{array}{l}\text { Can stopping rules and clear thresholds identify and/or minimize the perceived risks } \\
\text { of failures, to species and to institutions? }\end{array}$ \\
\hline \multicolumn{2}{|c|}{ Stakeholder and institutional support } \\
\hline Leadership & $\begin{array}{l}\text { Is there explicit policy guidance and leadership support for AM? Will stakeholders see } \\
\text { AM as an effective way to deal with uncertainty? }\end{array}$ \\
\hline Flexibility in decision making & $\begin{array}{l}\text { Is there sufficient management flexibility (and continuity) to incorporate new } \\
\text { information in revised experimental designs? }\end{array}$ \\
\hline Avoidance of taboo trade-offs & $\begin{array}{l}\text { Does the proposed AM design involve any trade-offs that might be considered taboo } \\
\text { by some stakeholders? }\end{array}$ \\
\hline Institutional capacity & $\begin{array}{l}\text { Are sufficient analytical skills available (staff or contractors) to design, evaluate, and } \\
\text { monitor AM plans? }\end{array}$ \\
\hline
\end{tabular}

We acknowledge the need for flexibility and agree that the more widespread application of decision-analysis techniques-and, more generally, adherence to the underlying principles and methods of sound decision making - will aid in the design and conduct of AM initiatives. However, we see the biggest obstacle to the more widespread, and successful, application of AM techniques as something different and, for better or worse, largely within the control of proponents of AM approaches. This obstacle has little to do with improved education for-or communication among - the participants in an AM process or better methods of evaluation. Instead, it exists mainly in the context of how the problems to which AM might be applied are identified and defined. What is needed, in addition to better communication and education and improved evaluation methods, is a set of conceptually sound yet practical criteria to help decision makers make thoughtful choices when it comes to the selection of problems that either are, or are not, appropriate for the application of AM techniques. Simply put, the problems facing AM may have less to do with the approach itself than with the indiscriminate choice of contexts within which it is applied.

\section{Criteria for Assessing the Viability of AM}

When considering an environmental management problem, we believe there are four topic areas that should be used to establish sensible criteria regarding its appropriateness for the application of AM techniques. These include (1) the spatial and temporal scale of the problem, (2) the relevant dimensions of uncertainty, (3) the associated suite of costs, benefits, and risks, and (4) the degree to which there is stakeholder and institutional support. Each of these criteria can be cast as questions to be posed by resource managers contemplating the use of an AM approach (see Table 1). These questions, and the responses they naturally imply, are intended to form a more defensible basis on which resource managers can systematically probe the pros and cons of various options for the selection and implementation of AM approaches.

In order to illustrate use of these criteria, the ensuing discussion employs four hypothetical but realistic casestudy scenarios that exhibit a range in complexity. They are realistic in the sense that they are grounded in actual examples for which resource managers and land-use planners have either considered or implemented an AM 
TABLE 2. Example case-study scenarios, presented in order of increasing level of complexity.

Problem 1. Tree fertilization

A field test to assess seedling growth response to alternative fertilization regimes on a set of cutblock regeneration sites. The study supports a classical experimental design including replication and randomization, allowing strong inferences to be made about causal relationships. The test sites are located within a large forest tenure area that has an approved long-term management plan in place and no significant jurisdictional/regulatory considerations nor stakeholder controversies.

Problem 2. Fisheries restoration

Assessing the choice of alternative restoration plans to meet federally mandated minimums for resident populations of salmonids downstream of a mid-sized hydroelectric dam near to a major metropolitan area. Two species of salmon, spring-run chinook and summer-run coho, have been declared as endangered under the terms of the Endangered Species Act. Developing a recovery plan will require a mix of both standard and innovative restoration actions designed to improve habitat quality and quantity. These actions are expected to require flow restrictions on water passing through the dam, reduced access to some upstream forest activities (to reduce siltation of spawning grounds), and limitations on further development of roads and housing projects in the area.

Problem 3. Wildfire fuels management

Assessing the efficacy of forest fuels management treatments to reduce wildfire risk in a wildland urban interface community. Fuels management alternatives include using mechanical fuels treatments, thinning, and prescribed burns. Developing the plan will require the direct involvement of provincial (or state) officials, local government, two forest companies holding tenure in the area, and community residents. Key issues to be considered are wildfire risks to community residents and to properties, smoke management and air quality, and the financial and socio-economic feasibility of alternative treatments.

Problem 4. Climate change and land-use planning

Assessing the effect of climate change on land use designations as part of a major regional land-use plan. The plan must indicate the location and extent of future protected areas (e.g., parks and biodiversity reserves) which, in turn, has implications for competing and complementary land uses (e.g., agriculture, forestry, urban development) as well as recovery and restoration activities in area rivers, lakes, and wetlands. Major climate change uncertainties include the effects of temperature changes on the health of fish populations, the effects of extended growing seasons on agricultural crops and tree growth and yield, increased threats from pests that might affect forest health, biodiversity, the possible influx of invasive plant species, and the influence of changing soil conditions on species compositions and distributions.

approach. As summarized in Table 2, these four cases include (at lower levels of complexity) a tree-fertilization application and a fisheries-restoration example and (as complexity increases) an assessment of wildfire fuels management and a regional land-use planning example.

\section{Spatial and temporal scale}

Most environmental management problems cover multiple geographic and temporal scales. Understanding the spatial and temporal dimensions of the decision context is an important starting point for probing opportunities to successfully apply AM. The basic question is whether it is reasonable to design experimental management regimes that might cover large geographic areas or extend many years - in some cases, decades - into the future.

Duration.-AM must account for the response time of parameters chosen as suitable end points for the resource-management problem. Support for AM initiatives is likely to be lower in cases where results of the proposed manipulation will take a longer time to become known. Holding other things constant, waiting a longer time for results means higher costs and a greater opportunity for contamination of the study design due to the influence of external factors. With respect to costs, evaluation schemes comparing alternative AM design options generally involve calculating the discounted sum of the expected annual net benefits (i.e., benefits minus costs), with annual values defined in terms of expected results based on probability-weighted hypotheses (Walters and Green 1997). Given the typical practice of using a positive discount rate (most often in the range 3\%-6\%) to estimate present-day equivalents, the value assigned to benefits or costs occurring in the near future (i.e., in $1-5$ years) is substantially greater that those occurring in the medium-term of far future (i.e., more than two or three decades hence).

To some extent the duration of a management strategy is a function of the problem context. In our simplest case, monitoring the growth response of seedlings to fertilization (Problem 1 in Table 2), the response time would be short (two or three years) and unproblematic from an experimental design point of view. On the other hand, monitoring the accumulation of forest fuels across alternative treatment regimes, as required in the wildfire fuels management case (Problem 3), might require decades. And taking into account the lag-time response of key landscape-level indicators of biodiversity to climate change (Problem 4), such as might be required to validate the selection of a protected area boundary within a land-use plan, suggests that very long timelines (several decades or more) would be required.

The duration of an AM plan is also a function of the selected design, and here AM proponents often have failed to do a careful job stating and/or analyzing their case (see Hilborn and Walters 1992). Consider Problem 2 , which could involve changes in water flows to encourage higher salmonid populations. An active AM approach (assuming baseline data of reasonable quality) might see three or four different flow levels, each held for up to four years, for a total duration of 12-16 years. Replication of these results would double this timeline. These are long time periods for any results-oriented management agency. One option is to consider setting the experiments up using a titration or step-down strategy, where rules are developed to help decide whether the results of the first or second trials are 
sufficiently strong that no further experimentation is necessary. Decision-analysis techniques are helpful in setting up this type of a priori analysis (i.e., by formally estimating the value of additional information (VOI) to be gained through additional trials) but they rarely have been used as supporting justification when proposing an AM plan.

Spatial complexity.-AM plans that involve large areas, such as Problem 2 (due to restrictions on other land uses) and the climate-change land-use problem (Problem 4), face numerous management hurdles due to the spatial extent of the associated impacts. From the standpoint of the ecological sciences, the types of broadscale questions often being addressed at this scale (e.g., the best location for a protected area as part of Problem 4) often preclude the use of replication and other important experimental-design elements; there is simply no comparable geographic area because of the extent of the AM-related consequences. This is significant, because learning requires a comparison to something, be it a control plot or a differently managed river or forest or landscape. While observational designs (Schwarz 1998) and retrospective studies (Smith 1998) offer a good deal of analytical support in such situations, these methods represent a compromise away from a "pure" experimental design.

A direct correlation also often exists between the geographic scale of the problem and the number of jurisdictions, policies, and stakeholders that must formally be taken into consideration. Not surprisingly, there are few examples of successful "true" experimental designs at the scale of watersheds or large ecosystems. What often happens, instead, is that AM initiatives are initiated on subsets of the problem (e.g., individual reaches or tributaries of a river) with few opportunities for the transfer of this learning to other areas or back to the overall management plan. Yet this lack of connection between subsets of a given AM plan need not be the case. If thoughtful choices are made about where to conduct assessments so that they focus on key uncertainties and can be "scaled up" so as to be applicable to larger areas, then AM initiatives can work well (for an example, see Bunnell and Dunsworth [2004]).

External effects.-A further consideration is controlling for background trends, including both other developments in the area that themselves create environmental changes and cumulative effects that result from other management initiatives taking place over the duration of a trial. Designing experiments, based on explicit hypotheses, that are sufficiently powerful to unravel the causal webs of interaction between management actions and ecosystem responses in the midst of large-scale environmental changes - what statisticians would call "nonstationarity" and others simply a "shock" - is no trivial matter. The sheer analytical complexity of designing AM experiments to cope with the confounding of results with trends external to the experimental treatment can be overwhelming. As a result, AM applications (especially in more dynamic management environments) are more likely to be successful when the management problem is tightly specified in terms of its temporal and spatial bounds.

From an AM-design perspective, anticipating the impact of external effects can add significantly to the complexity of an experimental design. Yet if this complexity is viewed as a blanket reason to forego learning opportunities through AM, then a host of potentially significant applications - involving questions such as those at the forefront of Problems 3 and 4-may be neglected and the scientific uncertainty associated with proposed strategies will largely be hidden from the view of decision makers. When the management environment is very active, and particularly if multiple resource- management agencies are involved in the study area, a better approach is to set up an AM design that recognizes complexity and has sufficient predictive capability to allow for a choice among management actions depending on the status and significance of anticipated external events. If this design capability is not possible-because of financial or temporal constraints, or due to a lack of predictive capability regarding the nature or timing of significant external events - then serious consideration should be given to restricting the scope of the trial so as to increase confidence in the anticipated ecosystem response.

\section{Dimensions of ecological uncertainty}

Dealing effectively with what ecological uncertainty implies for the design of environmental management plans is the core purpose of AM. Yet the term "uncertainty" covers a wide range of phenomena relating to the outcomes of a plan, the assumptions that underlie management interventions, the values associated with the anticipated consequences, and a variety of institutional responses. Resource managers who want to apply AM must carefully assess these various dimensions of uncertainty and the confidence which they and other participants (community residents, resource users, First Nations, academic scientists) have in the resulting assessments.

Structural uncertainty.- Structural uncertainty results when important relationships between ecological variables have not been identified correctly or when their functional form is not known with precision. Fervent AM supporters optimistically claim the surprises that may arise in such circumstances can provide some of the best opportunities for learning.

Unfortunately, the very notion of clearly documenting what we do not know as the basis for experimenting with valued and, in many cases, fragile ecosystems can pose a dilemma for any manager. It is hard to envision participants engaged in a land-use planning exercise that is addressing fundamental climate-change uncertainties who would willingly accept any experimental approach that could have 'surprising' adverse outcomes on an atrisk species, other conservation objectives, or even timber 
supply. Implementation of AM is difficult whenever significant surprise outcomes related to pre-identified structural uncertainties (and subject to multi-stakeholder examination) are possible. Before proceeding with an AM plan, therefore, managers must have some confidence in the level of resilience (i.e., the adaptability to change) that exists within both the ecological and social systems to be managed. Low levels of resilience must be considered carefully, regardless of AM's potential to reduce ecological uncertainty over time.

Parameter uncertainty.-A common point of contention in the design of AM plans is examination of the statistical uncertainty inherent in a proposed AM application. This dimension refers to the uncertainty associated with parameter values that are not known precisely but can be assessed and reported in terms of the likelihood or chance of experiencing a range of defined outcomes.

A variety of methods exist for representing probabilistic variables and model inputs, typically involving probability distributions (Morgan and Henrion 1990, Cullen and Frey 1999). When the underlying (ecological or causal) mechanisms are known, there can be a theoretical basis for selecting a particular distributional form; variables derived from multiplicative processes often approach a lognormal distribution, purely random processes often are represented by a Poisson distribution, and so forth. Yet even when such theoretical models are applicable, real-world conditions often lead to significant deviations. In some cases (particularly if data quality is high), parameter estimation techniques can be used to identify an appropriate distribution. In other cases (particularly if data quality is low or if there is substantial controversy or disagreement among experts), there is often no substitute for expert-judgment elicitation techniques. In such cases, technical experts might (for example) be asked to estimate the 90 percent confidence intervals for a calculated expected value, such as the maximum seedling growth in five years (e.g., Problem 1) or the expected juvenile salmon biomass (e.g., Problem 2).

AM seeks to apply the techniques of formal scientific investigation so as to reduce parameter uncertainty through the design of experimental trials or effective monitoring regimes that will be capable of refining or redirecting implementation methods. In the case of assessing alternative forest-fertilization regimes, the opportunity to develop statistically powerful experimental trials is readily evident. Unfortunately, the ability to successfully meet the strict requirements for randomization, replication, and representation lessens with both the number and scope of the uncertainties that must be probed. Consider the case of the land-use plan (Problem 4): developing an experimental or monitoring design capable of dissecting the interacting effects of changes induced by climate change on forest growth rates, natural disturbances, and species composition using end points that include timber supply and biodiversity conservation would be a monumental task. This suggests that scientists must be realistic about the ability of AM experiments to reduce uncertainty, rather than simply develop a better understanding of it, and that careful screening of uncertainties is required to distill which sources of uncertainty are thought to matter the most from the standpoint of stated management objectives and feasible alternatives.

Stochastic uncertainty.-Stochasticity, or variation due to pure chance and unrelated to systemic factors, is a particular form of uncertainty that requires special attention in the design of AM initiatives. The problem from a design perspective is that inherent randomness, associated with many aspects of nature, is irreducible in principle. Stochastic uncertainty thus affects the design of AM experiments to the extent that outcomes are dependent on the frequency of, and control over, an unpredictable yet important triggering event or condition. Consider the assessment of fuel-management treatments in Problem 3. While it is possible in theory to apply most of the tools for a powerful statistically designed experiment, the ultimate outcome-understanding the efficacy of treatments in reducing wildfire impacts - is dependent on experiencing a wildfire itself. However, a wildfire may occur partway through a multiyear treatment program or 50 years afterwards or not at all; it may be very intense or slow; and it may have a wide range of different effects on the forest (e.g., it may affect only tree crowns or burn surface debris and soils). Such an uncooperative (from an AM standpoint) natural event may "test" certain treatment areas and not others.

Under these circumstances, then, the question becomes: To what extent will managers be able to attribute identified outcomes (e.g., a low-intensity fire within a certain treatment area or the absence of a destructive fire altogether) to a specific AM plan? If managers have little or no confidence in their ability to provide a positive response, then the added value of conducting experimental trials (in contrast to passive AM or even simple "best-guess" management) may be minimal. Thus, experimental AM may be an unreasonable concept when the resolution of key sources of uncertainty relies on low probability, randomly triggered, and highly variable events. One response to stochastic uncertainty could be to expand the duration of the AM treatment, since randomness will tend to "settle out" over time and thus make it easier to separate signal from noise. However, such a strategy may conflict with other objectives such as cost or external effects and would also need to be balanced with a temporal scale tolerated by managers and key stakeholders.

Confidence in assessments. - A final important dimension of ecological uncertainty is the degree of confidence in assessments held by scientists and other participants. If the level of uncertainty is high (for any of the reasons discussed above), then the use of AM may be inappropriate because the results of planned experi- 
ments will not be interpretable. Moreover, if very little is known, then it may be impossible to develop testable hypotheses or to separate the effects of experimental manipulations from external influences without the benefit of additional data (e.g., from baseline field studies, modeling, etc.).

However, it is unclear in many cases if the lack of confidence in assessments is brought on by real uncertainty surrounding the system or if it is the product of limited precision across the sciences. One response to this dilemma is to import information from another ecologically comparable area (at least with respect to key dimensions of the problem under consideration) about which substantially more is known. Another response is to make use of expert-judgment techniques, based on the methods of decision analysis, which can help to clarify assessments of confidence in two ways: they can help to make assessments of confidence explicit, for example by moving from verbal to quantitative statements of uncertainty and thus overcoming linguistic imprecision, and they can help by making explicit any differences between experts. Formal techniques for ascertaining the level of confidence in assessments are well defined (Morgan and Henrion 1990, Keeney and vonWinterfeldt 1991) and analytical approaches to explicitly express the degree of confidence in judgments continue to improve. For example, methods for documenting a "traceable account"-i.e., a formal record of the lines of evidence used and the means of reconciling any differences among them-have become more common (Moss and Schneider 2000). More recently, van der Sluijs et al. (2005) and others have developed formal approaches to documenting the pedigree of information sources as a semi-quantitative rating of reliability. These advances are encouraging and should be promoted further when considering the implementation of AM, as should the general use of formal expertjudgment elicitations (Gregory and Failing 2002).

Nonetheless, numerous writers on the topic of AM have pointed out the inherent difficulties associated with bridging the gap between scientists, managers, and stakeholders on the topics of confidence and credibility. Walters (1997), for example, has chastised scientists who promote research self-interests, political decision makers who blame inaction on the need to first resolve uncertainties, and stakeholders who focus on a single uncertain ecological value. A skilled participant can nearly always spin issues of uncertainty management in creative and self-serving ways. In our fuels- management case study, for example, individuals opposed to prescribed burns due to misperceptions about their ecological risks can emphasize a lack of confidence in estimates of smoke impacts on the elderly or aesthetic effects on tourism to the extent that they feel these arguments will help to win over a larger - and similarly opposed-audience. While this type of strategizing can occur at almost any scale of AM application, its likelihood mounts as the uncertainties become more profound, the consequences more severe, and selfinterests increasingly threatened.

\section{Evaluating costs, benefits, and risks}

Many AM approaches fail or are abandoned because proponents do not fully understand, or have not taken the time to identify, the targets that they seek to achieve. Accurate predictions of future costs, benefits, and risks that will result from an AM plan hinge upon the careful specification of its often wide-ranging consequences. To this end, the basic framework for evaluating the costs, benefits, and risks of adaptive-management options should be no different from that required for any other resource-management initiative: first clearly define management objectives (which can broadly be characterized as controlling costs, maximizing benefits, and reducing risks) and then use these multiple objectives to evaluate a plausible range of alternatives, while taking into account key uncertainties regarding both consequences and likely institutional responses. Added to this basic framework is the requirement to state a range of possible hypotheses about the response of the natural system, and to evaluate design options based on the probability of each hypothesis being correct.

Specifying benefits and costs.-Identifying the benefits of AM plans begins with all the standard problems (How will changes in habitat quality affect future numbers of a key species? How will changes in land prices over the next 30 years affect population densities near to a protected area?) but adds to these the problems of addressing multiple trials that will achieve their results with varying probabilities of success. Simply collecting the information required to complete each of these evaluations can be particularly difficult, and time consuming, when considering alternative AM proposals. With active AM plans, for example, the plausible range of values for the outcomes of interest need to be estimated for each of several hypotheses about the prevailing states of nature (Gregory et al. 2006). Small wonder that decision makers often need (and do not always receive) help in deciding between a single nonexperimental plan (i.e., passive AM, with monitoring for the key sources of uncertainty and flexibility in future management options) and an experimental program of comparative trials (i.e., active AM, involving several explicit experimental treatments).

Technical specialists who work over many months or years on an experimental regime often feel that their design is close to ideal in the sense that all possible influencing factors have been taken into account. In our experience (as outside analysts, called in to evaluate such plans), we have yet to see the perfect strategy. This point is not intended to confuse good decision making, which is within the control of managers, with the success of outcomes, which - because of factors such as variability and stochastic uncertainty - will remain, to some degree, outside their control. Instead, the conclusion is that the 
predictive capacity of study hypotheses is generally less than anticipated - often substantially so.

Within the context of these general difficulties in anticipating the benefits and costs of AM plans, there are two issues of particular concern. The first is the need to weigh the impact of potential opportunity costs. As discussed above, long time lines can make it difficult for managers to take other actions in the same geographic area or affecting the same resources. To the extent that other beneficial actions (e.g., one-time-only habitat enhancements with a short turn-around time) are postponed in order to preserve the clarity of experimental results, this represents an opportunity cost (associated with foregone options) that might not be possible to define at the inception of the AM initiative. The second issue arises when definitive actions may need to be taken sooner than expected due to institutional or political reasons, which could (in the extreme) result in the midcourse termination of an AM plan. Either way, unanticipated changes in the experimental design will have the unfortunate effect of decreasing the relevance of a priori evaluations and will make it more difficult, if not impossible, to interpret with sufficient accuracy the results of trials or ongoing monitoring.

Magnitude of effects.-It is perhaps obvious, but often (it seems) overlooked, that the results of an AM trial need to be measurable, as distinct from base-line conditions and background noise. From a practical standpoint, this may influence the design of an AM initiative and the development of guiding hypotheses. For example, in Problem 2 it is generally much easier to achieve clearly measurable benefits in the form of increases in the quantity of habitat than it is to achieve measurable increases in habitat quality. The ease with which one attribute (such as quantity) can be measured should not preclude a focus on other relatively less tractable improvements (such as habitat quality) as part of an AM plan. In-depth discussion of the measurability of impacts during AM planning emphasizes the need for creative techniques to develop effective measures of anticipated changes in objectives. Decision-analysis techniques again provide a good source of ideas, including an emphasis on constructed indices that can facilitate the creation of problem-specific measures for key ecological and community variables (Keeney and Gregory 2005).

The results of an AM manipulation also need to be sufficiently large for them to matter. If the magnitude of anticipated results is too small, then the expected benefits simply will not count: the change will fall below some threshold measure of the least-significant-impact magnitude. Stated differently, if a change across the range of probabilistic improvements in a key evaluation criterion is not expected to lead to a management change, then the proposed treatments may be scientifically interesting but practically insignificant. This point has three elements. First, measures of benefits need to have some appeal to a broad audience; consider, for
Problem 2, the difficulty that non-technical audiences might have interpreting the significance of changes in a "smolt to spawner" ratio. Second, the consequence (i.e., the before-after or with-without change in a measure) must be sufficiently large that it is coded as being significant, that is, as making a difference to managers and other key stakeholders. Third, explicit hypotheses need to be developed so that the significance of responses can be assessed in advance (rather than on an ad hoc basis) and so that links to management actions can be made efficiently.

Multiple objectives.-Adaptive management (and environmental management in general) would be far simpler if there were only one objective of concern. For example, fisheries-recovery actions could be taken to maximize population abundance, or silvicultural activities could seek solely to maximize yield in a timber supply area. In actuality, management activities must take into account multiple objectives: fisheries-recovery actions need to address other ecological (e.g., species diversity) and economic (e.g., commercial-harvest interests) concerns, as do silviculture treatments (e.g., minimize use of harmful herbicides, provide high-quality wood, and so forth). Rarely is there a single, dominant planning objective to serve as the focus of experimentation, such as in our simplest case of conducting fertilization trials (Problem 1). More often there are multiple objectives that need to be addressed, and tracked over time, using both formal and informal assessment methodologies.

When assessing the relative reduction of wildfire hazard among prescribed-burn and mechanical-thinning treatment approaches, as in the case of Problem 3, the core experimental hypotheses may be conceived quite narrowly. However, the evaluation of design options and the implementation of the experiment itself must explicitly address other objectives including the risk posed to community assets, economic cost, employment implications, and smoke-related health and aesthetic concerns. These other objectives also must be addressed when selecting among management options (Ohlson et al., in press). In short, when considering the appropriateness of AM it is not enough that a given plan will further scientific knowledge. Instead, the results need to matter in the sense of making a substantial difference in the context of the multiple objectives important to decision makers, who otherwise have little reason to allocate scarce public funds to an AM (or any other) approach.

Broadening the scope of AM requires that proponents design a plan so that it clearly addresses trade-offs among objectives, because these are likely to be important to stakeholders for whom ecological interests are of lower priority than related economic, cultural, or social concerns. For example, the biodiversity benefits of a fuels-management AM plan may make a difference to ecologists but not to homeowners; to capture homeowner support, links may need to be drawn from a 
healthy forest to reduced fire danger and, in turn, to higher economic values for properties. Scientists who work hard to develop an AM plan may view such additions as lessening the purity of their planned experiment, but we are not sympathetic: making ties to other objectives not only helps to develop support but also explicitly recognizes the multiplicity of values that are at play.

Perceived risks of failure.-Typically, an AM approach will suggest either multiple interventions at different sites (so that results can later be compared) or at different times (so that a choice can later be made favoring the preferred trial). The need to compare multiple trials means that some will be more successful than others, which can lead to the characterization of less successful trials as failures, at least in a relative sense. In such cases the perspective of those who organize the experiment can be quite different from that of those who, for example, live or work in or have concerns about the area where a less-successful trial was run; agency scientists may code as successful the same AM experiment that others code as a failure. Although obvious prescriptions follow, such as communicating the rationale for the AM strategy clearly with all potentially affected parties, there is little evidence that proactive public-outreach efforts as part of AM will dampen critiques of the method.

Yet AM initiatives are fundamentally about learning, and as choices are made along the continuum from passive to active AM - presumably in hopes of increasing learning - there is also a greater likelihood that what is tried will fail. For most technically trained scientists, "failure" is a relative term in the sense that it is viewed against the alternative management options. For example, if the risk of extinction is high and action is urgently needed to save a species, then experimentation guided by the principles of AM may well make sense despite the uncertainty over outcomes. In this case, the perceived risk of failure is likely to be lower with the AM initiative than without. Other stakeholders, however, may not pay sufficient attention to the default (do nothing, or do what seems best) option and, if population numbers decline, this so-called "failure" might well be blamed on the adoption of an AM approach.

Nevertheless, fear of failure should not necessarily lead to the adoption of monitoring over passive AM, or to passive over active AM. Instead, managers need to work closely with stakeholders to define their concerns and assess their risk tolerance, then develop mechanisms such as stopping rules that, when built into the design of AM plans, clearly identify thresholds and have the power to halt an experiment should these values be threatened.

\section{Stakeholder and institutional support}

Lee (1993) clearly articulates that institutional support is required to successfully undertake AM. At a minimum, there needs to be an awareness among decision makers that reductions in uncertainty are a necessary focus of management actions and require specific policy guidance. This support needs to be relatively stable: continuity in support is critical so that resources for an adaptive management plan are not withdrawn part-way through an experimental or monitoring plan. There also needs to be a clear connection between the activities of biological scientists and the goals and objectives of key decision makers. In our experience the successful implementation of AM also requires several other factors: leadership to guide stakeholders in understanding key benefits of the AM plan, the ability to be flexible in responding to a range of management options, avoidance of concerns that might prevent AM trials, and a high degree of competence among technical staff.

Leadership.-For discussions about AM trials to be productive, there needs to be a recognition among those who design, and those who make decisions about, the proposed experiments and monitoring plans that a specific focus on reducing ecological uncertainty will lead to improved environmental-management strategies. For implementation of an AM plan to be successful, there also needs to be strong leadership that will guide the discussions among stakeholders and address their concerns about a management strategy that is explicitly experimental in nature. This is manifested in an obligation to demonstrate the value of using an adaptive approach rather than some other method (Gregory et al. 2006). Lee (1993) and others (e.g., Westley 2002) have emphasized that this is challenging, largely because it requires framing the "policy-oriented" learning that is possible through AM in terms that recognize the world of real politics as well as the cognitive and behavioral limitations that individuals operate under when making choices about complex and novel options. Of course, one perspective on the experimental nature of AM is to emphasize that all management actions, including the option of doing nothing, also are experimental in that they involve the implementation of actions with uncertain consequences. In this sense, AM plans are simply more explicit about the attendant uncertainty.

At a process level, the need for leadership becomes even more pronounced when the group responsible for making a decision body is made up of a large, diverse group of stakeholders. Whereas agreement might easily be reached among agency scientists and other technically trained participants, the expansion of participants to include community members, other resource users, and First Nations/Native Americans can increase the potential for conflict - and the apparent need for compromise, resulting in a watered-down version of an AM experiment. Stakeholders are likely to feel uneasy whenever key ecological information is unknown or contested, particularly to the extent they view themselves as stewards of the public trust and, thus, responsible should a decision made today have unforeseen adverse consequences in the future (McDaniels and Gregory 2004, Froschauer and Arvai 2006). A focus on adaptive 
learning provides a way to move forward in the face of limited information and unfamiliar trade-offs because decisions made today will be revisited in the future, once more has become known. Thus, an AM strategy can change one-time decisions into iterative, sequential decisions with opportunities for later refinement and adjustment, thereby easing concerns about later being held responsible for a consequence viewed as undesirable (Failing et al. 2004).

Flexibility in decision making.-Proponents of AM need to think about whether there is sufficient flexibility within the broader regulatory framework to respond to the new information that AM monitoring or experimentation may provide. Consider getting 10 years into a fuels-management program and discovering that prescribed burning is offering significantly better performance from a risk-reduction standpoint than expected. Would management agencies allow for a ramping up of this activity?

Of course, the question of flexibility in response is a two-sided undertaking. Institutions do need to have a willingness to be flexible and to act in response to new information, assuming that trade-offs across objectives are addressed; in the previous example, for instance, improved performance from a risk-reduction standpoint would need to be weighed against the predicted outcomes associated with increased burning as they relate to other objectives such as maintaining suitable air-quality levels or meeting certain ecological targets. Likewise, scientists and others who aid in the design of AM plans also must, a priori, develop clear protocols for which adjustments are needed, including the definition of clear triggers (what thresholds or results should initiate a change?) and management responses (in the event a threshold is reached, which is likely to be the appropriate management change?).

Avoidance of taboo trade-offs.-Managers contemplating adoption of an AM approach must also ask whether the proposed experiments might create unacceptable or highly controversial risks, often characterized in the literature as taboo trade-offs (Fiske and Tetlock 1996) or protected values (Baron and Spranca 1997). Such concerns are marked by characteristics such as quantity insensitivity (it does not matter how much of something is affected-even a little is too much). Oftentimes, taboo trade-offs are accompanied by judgmental paralysis; the moral obligation to "do the right thing" cannot be reconciled with the need to make trade-offs across objectives that seem equally important (e.g., the desire to simultaneously protect both human and environmental health). Previous authors, for example, have highlighted the conundrum posed when the potential outcomes of management experimentation may impact sensitive species at the expense of other important social objectives (e.g., Walters 1997). Of course, the existence of a sensitive or endangered species often is the reason for consideration of an AM initiative, which poses an interesting intellectual and legal quan- dary: AM may be most difficult to implement in precisely those circumstances where it is most needed.

Consider, for example, the case of the interface fuelsmanagement problem (Problem 3) where "experimenting" suggests that some communities or areas might knowingly be exposed to higher wildfire risks than others. In this case, once the risk aversion of participants (including elected officials) comes into play, the frequent result is a weaker experimental design wherein proposed interventions may not be significant enough to trigger a meaningful ecological response. Simply put, once taboo trade-offs arise as part of the evaluation of consequences, the feasibility of AM becomes severely limited unless a creative way is found to address these concerns. Helpful methods do exist (Gregory 2002), but (as noted below) the question is whether managers are knowledgeable and comfortable in using them.

Institutional capacity.-Any environmental management plan is only as good as the capacity of the implementing institution to deliver on its promises. In this regard, there are some special problems with respect to the adoption of an AM plan. The most obvious has to do with the training of managers. As conveyed by a cursory glance through the applied AM literature (e.g., Walters and Green 1997, Failing et al. 2004), the required level of statistical and analytical sophistication is quite high. Earlier discussions noted the need for use of VOI (value of added infrastructure) studies (see Duration, above) and methods to explicitly address difficult trade-offs; other common techniques include Monte Carlo simulations and expert-judgement elicitations. A common problem is that these skills, needed to design statistically valid AM treatments, seldom exist inhouse and often require additional contract resources.

In those cases where individuals with the required expertise are on staff, the claims on their time are likely to be severe. For example, AM plans may require annual (and in many cases, more frequent) assessments of ongoing trials followed by decisions concerning whether sufficient new information has been generated that something other than current practices should continue. Nor is it a straightforward matter to hire consultants or academics to lend a hand; in contrast to many of the other skills held by ecologists or biologists or planners or policy analysts, relatively few individuals have been trained in the statistics or methods needed to successfully design and evaluate AM plans.

\section{DisCUSSION}

Not all environmental-management problems require significant learning in order to reduce ecological uncertainty; instead, many management actions present themselves as obvious and common-sense choices, so that the value of additional reductions in uncertainty is negligible. However, even if an environmental-management problem justifies attention to the objective of reducing uncertainty and if the resources are available to attempt some form of active AM, we still suggest that 


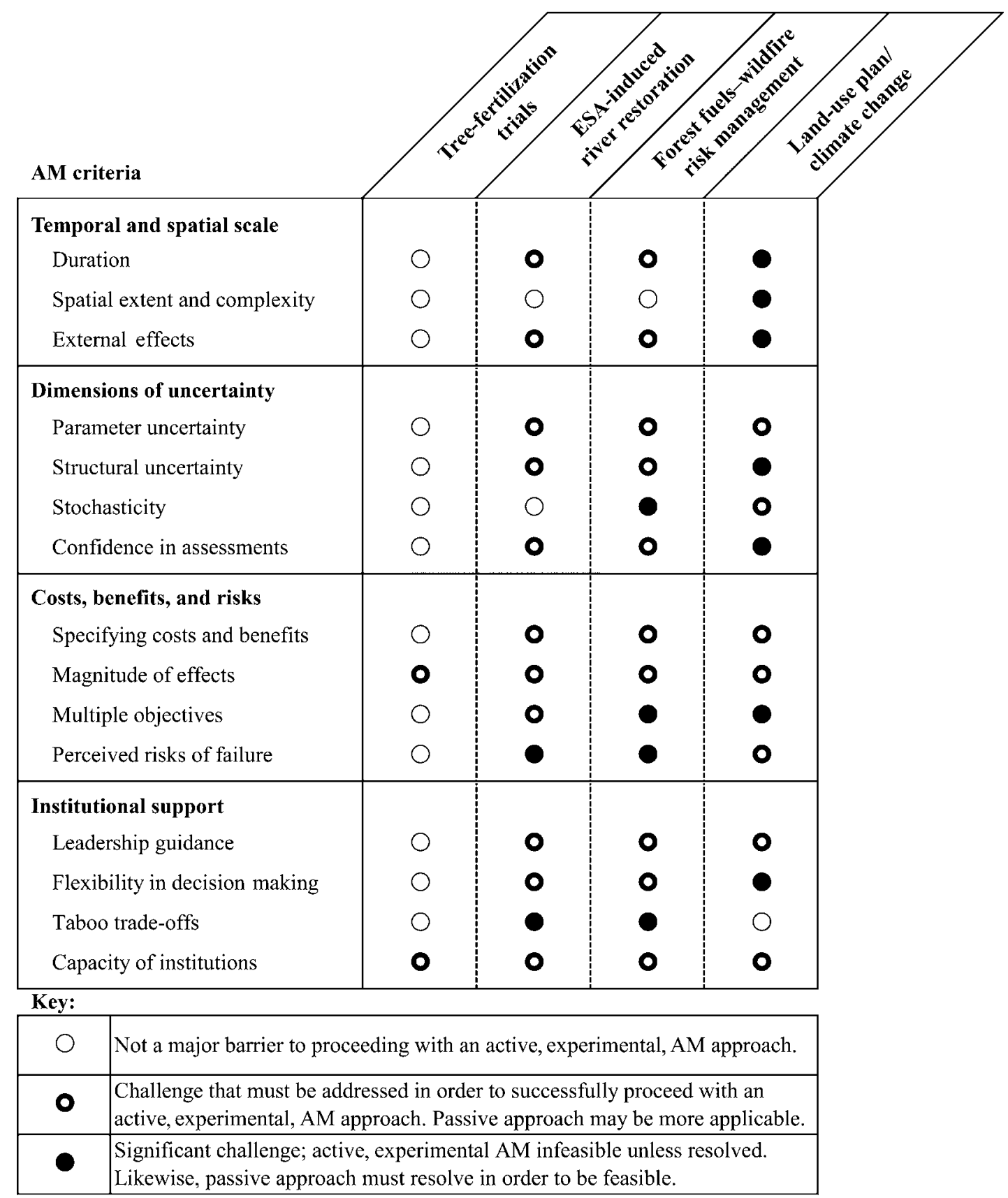

FIG. 1. Application of the proposed adaptive-management (AM) criteria to four hypothetical but realistic case-study example problems.

proponents consider the criteria outlined in the preceding discussion.

Fig. 1 presents an overview of the application of the criteria to our hypothetical planning problems using a simple three-tier rating system that distinguishes among aspects of environmental-management problems in terms of (a) those that will not prove difficult for AM applications, (b) those that will present acceptable challenges, and (c) those that will present significant challenges, particularly to the application of active, experimental, AM approaches.

With Problem 1, assessing tree-fertilization alternatives, there should be no significant impediments to proceeding with a comprehensive, active, AM approach. In particular, the opportunity to develop a statistically powerful suite of experimental trials is readily evident. The only challenges that would need to be addressed would be ensuring that adequate staff time and skills 
were in place to support the initiative, and that the results of the trials could be shown to have an effect of sufficient magnitude so as to justify the investment.

The assessment becomes more complicated in Problem 2, the ESA-induced river restoration case. Here significant, yet not insurmountable, challenges to an active, experimental, AM approach exist in terms of (a) designing statistically powerful experiments capable of discerning external effects and effectively considering issues of duration (i.e., using titration designs), (b) articulating all the costs, benefits, and risks of alternative experimental and non-experimental management plans, and again (c) ensuring that sufficient staff capacity and institutional flexibility exist. The most significant hurdles, however, relate to the potential existence of taboo trade-offs in the form of experimenting with endangered species and the related (perceived) risks associated with failure. Even a passive AM approach might be significantly challenged by the requirement to get resource managers to explicitly state and agree upon the hypotheses related to the structural uncertainties that underlie the river-restoration plan.

Nonetheless, Problem 2 seems like a good candidate for implementation of a passive AM approach for at least the most significant management challenge, flow regulation through the hydroelectric dam. Managers could develop a best-guess operating scheme based on stated hypotheses regarding the potential for recovery of endangered salmon. They could also develop, in advance, the set of triggers and actions that would be implemented based on intensive monitoring results. Problem 2 might also provide an opportunity to implement a limited-scope active AM approach on an important sub-problem. For example, assume that suitable sub-watersheds exist to enable a paired experimental study of alternative forest or housing-development impacts on hydrology and water quality. In time, it is conceivable that well-planned active AM on such subproblems could be scaled up to influence broader forest management and development regulations throughout the region.

A very different set of challenges face the pursuit of an active AM approach for Problem 3, the wildfire fuelsmanagement case. As described above (see Criteria for assessing...: Dimensions of ecological uncertainty: Stochastic uncertainty, above), the key triggering event necessary to ultimately learn about the efficacy of alternative management schemes, in this case a wildfire, is a low-probability random phenomenon. We believe that it is unrealistic to both design an active AM approach as an overall guiding framework, and to expect stakeholders to agree to it up front, when significant challenges exist in the form of such stochastic uncertainties. Further, in this problem there is simply no getting around the need to address multiple objectives as part of the planning process. For example, smokemanagement considerations must be taken into account, as well as the potential risk to community assets from escape fires, if one wants to implement a program of prescribed fires near a community. These are very real and tangible objectives that must be integrated into the evaluation of experimental designs, for all of the multiple-objective problems noted above (e.g., weighing long-term reductions in wildfire probability and habitat improvement against short-term smoke-management objectives and significant front-end financial costs).

Problem 3 does, however, represent an importantenough problem that a commitment to the use of a passive AM approach might be warranted. As discussed, managers could develop a single-option management approach that might involve the combined use of mechanical thinning and prescribed burning across the landscape in a manner justified by stated hypotheses. They could similarly develop, in advance, a set of triggers and actions that may, admittedly, have to wait for the results of a wildfire, or be confounded if one were to occur prematurely. Nonetheless, the up-front effort at stating hypotheses should provide for some degree of learning over time and help to target monitoring so as to lead to the greatest reductions in uncertainty.

For Problem 4, land-use planning under climate change, the temporal and spatial scale issues alone are enough to eliminate a comprehensive active AM approach. It is simply inconceivable to envision a large-scale and long-term experimental design aimed at probing the preferred location of protected areas and areas managed for forestry and agriculture. Significant challenges would need to be overcome in order to implement even a passive AM approach that could form the basis of a long-term, flexible management plan. In past examples where AM has been attempted as a guiding management philosophy at such a large scale, such as the Columbia Basin or the Florida Everglades, there was a distinct lack of explicitly stated scientific hypotheses, monitoring triggers, and management implications, and no clear indication of whether the policy framework would be sufficiently flexible and responsive to adapt to learning over time. Experience has shown that this type of management context is a prescription for costly implementation failures.

\section{Conclusion}

Despite its obvious attractions-Who would not want to advocate a plan that promises reductions in uncertainty through learning?-AM is not an approach to be adopted without forethought and careful analysis. Some of the barriers and ecological complexities discussed in this paper pose significant challenges that must be addressed if proponents desire to defensibly select and implement an AM approach. Others, such as the influence of external effects or the existence of lowprobability, stochastic triggering events, unfortunately can simply be unsolvable in some cases. The trick is to incorporate into experimental designs those significant factors that can be anticipated and to recognize when 
conditions are, and are not, conducive to the choice of $\mathrm{AM}$ an as approach to environmental management.

The four categories of criteria discussed in this paper are intended to provide an explicit basis for making the decision about whether to choose an AM approach. Modification in specific circumstances will of course be necessary; these criteria, at best, will provide a starting point for the development of more focused site- or program-specific criteria. Our assertion, as decision scientists with an interest in the design of sound environmental plans and policies, is that having explicit criteria at the beginning of deliberations about environmental-management options (which may include AM) is better than the typical current practice, which involves coming up with ad hoc criteria to justify a suite of actions partway through the development of a management plan.

This emphasis on proactive criteria is also intended to help distinguish true AM initiatives from imposters. Adaptive management, as currently invoked, is far too often used simply as a euphemism for environmentalmanagement plans that admit to the need for learning in the face of ecological uncertainty but lack the other components - attention to spatial and temporal scale, structured hypotheses that acknowledge different types of uncertainty, a design that addresses evaluation needs, and attention to institutional and stakeholder supportthat are necessary for the design of an effective and defensible AM plan.

Finally, this discussion is intended to help lay the groundwork for a more informed consideration of AM by both expert and nonexpert stakeholders. Increasingly, discussions about potential adaptive-management options involve a wide range of participants, including many with little or no training in ecology or biology. In our opinion, this is entirely appropriate: the decision to undertake an AM strategy is appropriately placed within a broader policy context due to the important economic and social as well as biological implications. In this context, the value of AM as a guiding philosophy should not provide an excuse for its proponents to neglect the tough work required to come up with a strong and fully specified management plan. The failure to do so neglects both the promise of adaptive management and the social contract within which it is undertaken.

\section{ACKNOWLEDGMENTS}

We thank Lee Failing and Basil Stumborg for many insightful discussions about adaptive management and Andy Haas, Paul Higgins, and Carl Walters for helpful comments on an earlier draft of this paper. Financial support was provided by the U.S. National Science Foundation through Award SES0350777 to The Ohio State University and to Decision Research.

\section{Literature Cited}

Baron, J., and M. Spranca. 1997. Protected values. Organizational Behavior and Human Decision Processes 70:1-16.
Bunnell, F., and B. Dunsworth. 2004. Making adaptive management for biodiversity work - the example of Weyerhaeuser in coastal British Columbia. The Forestry Chronicle 80:37-43.

Clark, W. 1980. Witches, floods, and wonder drugs: historical perspectives on risk management. Pages 287-318 in R. Schwing and W. Albers, Jr., editors. Societal risk assessment: How safe is safe enough? Plenum Press, New York, New York, USA.

Cullen, A., and H. Frey. 1999. Probabilistic techniques in exposure assessment. A handbook for dealing with variability and uncertainty in models and inputs. Plenum Press, New York, New York, USA.

Failing, L., G. Horn, and P. Higgins. 2004. Using expert judgment and stakeholder values to evaluate adaptive management options. Ecology and Society 9(1):13 [online].

Fiske, A., and P. Tetlock. 1996. Taboo trade-offs: reactions to transactions that transgress spheres of justice. Political Psychology 18:255-297.

Folke, C., S. R. Carpenter, B. H. Walker, M. Sheffer, T. Elmqvist, L. H. Gunderson, and C. S. Holling. 2004. Regime shifts, resilience, and biodiversity in ecosystem management. Annual Review of Ecology and Systematics 35:557-581.

Froschauer, A., and J. Arvai. 2006. Good decisions, bad outcomes: the interaction of process and outcome in lay evaluations of decision quality. Journal of Risk Research, in press.

Gregory, R. 2002. Incorporating value trade-offs into community-based environmental risk decisions. Environmental Values 11:461-488.

Gregory, R., and L. Failing. 2002. Using decision analysis to encourage sound deliberation: water use planning in British Columbia, Canada. Journal of Policy Analysis and Management 21:492-499.

Gregory, R., L. Failing, and P. Higgins. 2006. Adaptive management and environmental decision making: a case study application to water use planning. Ecological Economics 58:434-447.

Gunderson, L., and C. S. Holling, editors. 2002. Panarchy: understanding transformations in human and natural systems. Island Press, Washington, D.C., USA.

Gunderson, L., C. S. Holling, and S. Light, editors. 1995. Barriers and bridges to the Renewal of Ecosystems and Institutions. Columbia University Press, New York, New York, USA.

Halbert, C. L. 1993. How adaptive is adaptive management? Implementing adaptive management in Washington State and British Columbia. Reviews in Fish Biology and Fisheries 1:261-283.

Holling, C. S., editor. 1978. Adaptive environmental assessment and management. John Wiley and Sons, New York, New York, USA.

Keeney, R., and R. Gregory. 2005. Selecting attributes to measure the achievement of objectives. Operations Research 53:1-11.

Keeney, R., and D. von Winterfeldt. 1991. Eliciting probabilities from experts in complex technical problems. IEEE Transactions on Engineering Management 3(3):191-201.

Lee, K. 1993. Compass and gyroscope. Island Press, Washington, D.C., USA.

McConnaha, W. E., and P. J. Paquet. 1996. Adaptive strategies for the management of ecosystems: the Columbia River experience. American Fisheries Science Symposium 16:410 421.

McDaniels, T., and R. Gregory. 2004. Learning as an objective within a structured risk management decision process. Environmental Science and Technology 38:1921-1926.

McLain, R. J., and R. G. Lee. 1996. Adaptive management: promises and pitfalls. Environmental Management 20(4): $437-448$. 
Morgan, G., and M. Henrion. 1990. Uncertainty. Cambridge University Press, New York, New York, USA

Moss, R., and S. Schneider. 2000. Uncertainties in the IPCC TAR: recommendations to lead authors for more consistent assessment and reporting. Pages 33-51 in R. Pachauri, T. Tanaguchi, and K Tanaka, editors. Guidance papers on the cross cutting issues of the third assessment report of the IPCC. World Meteorological Organization, Geneva, Switzerland.

Ohlson, D., T. Berry, R. Gray, B. Blackwell, and B. Hawkes. In press. Multi-attribute evaluation of landscape-level fuel management to reduce wildfire risk. Forest Policy and Economics. [doi: 10.1016/j.forpol.2005.01.001]

Peterman, R. M., and C. N. Peters. 1998. Decision analysis: taking uncertainties into account in forest resource management. Pages 105-128 in V. Sit and B. Taylor, editors. Statistical methods for adaptive management studies. Land Management Handbook 42. B.C. Ministry of Forests, Research Branch, Victoria, British Columbia, Canada.

Rogers, K. 1998. Managing science/management partnerships: a challenge of adaptive management. Conservation Ecology 2:2 [online].

Sainsbury, K. 1991. Application of an experimental approach to management of a tropical multispecies fishery with highly uncertain dynamics. ICES Marine Science Symposia 193: 301-320.

Schwartz, C. J. 1998. Studies of uncontrolled events. Pages 19 40 in V. Sit and B. Taylor, editors. Statistical methods for adaptive management studies. Land Management Handbook 42. British Columbia Ministry of Forests, Research Branch, Victoria, British Columbia, Canada.

Smith, G. J. 1998. Retrospective studies. Pages 41-54 in V. Sit and B. Taylor, editors. Statistical methods for adaptive management studies. Land Management Handbook 42. B.C. Ministry of Forests, Research Branch, Victoria, British Columbia, Canada.

Taylor, B. S., L. Kremsater, and R. Ellis. 1997. Adaptive management of forests in B.C. Report for the Forest Practices Branch, Strategic Policy Section, British Columbia Ministry of Forests. Queen's Printer, Victoria, British, Columbia, Canada.

van der Sluijs, J., M. Craye, S. Funtowicz, P. Kloprogge, J. Ravetz, and J. Risbey. 2005. Combining quantitative and qualitative measures of uncertainty in model-based environmental assessment: the NUSAP system. Risk Analysis 25: 481-492.

Walters, C. 1986. Adaptive management of renewable resources. McMillan, New York, New York, USA.

Walters, C. 1997. Challenges in adaptive management of riparian and coastal ecosystems. Conservation Ecology 2:123.

Walters, C., and R. Green. 1997. Valuation of experimental management options for ecological systems. Journal of Wildlife Management 61:987-1006.

Walters, C., and C. S. Holling. 1990. Large-scale management experiments and learning by doing. Ecology 71:2060-2068.

Walters, C., L. Gunderson, and C. S. Holling. 1992. Experimental policies for water management in the Everglades. Ecological Applications 2:189-202.

Westley, F. 2002. The devil in the dynamics: adaptive management on the front lines. Pages 333-350 in L. Gunderson and C. S. Holling, editors. Panarchy: understanding transformations in human and natural systems. Island Press, Washington, D.C., USA. 